\title{
Evaluation of complications developing during and after transvaginal ultrasound - guided oocyte retrieval
}

\author{
Selin Özaltın ${ }^{1}$, Serkan Kumbasar², Kadir Savan ${ }^{1}$ \\ ${ }^{1}$ Department of Obstetrics and Gynecology, Süleymaniye Research and Education Hospital, Istanbul, Turkey \\ ${ }^{2}$ Department of Obstetrics and Gynecology, GOP Taksim Research and Education Hospital, Istanbul, Turkey
}

\begin{abstract}
Objectives: To investigate the complications of the oocyte retrieval procedure currently used in in vitro fertilisation.

Material and methods: We examined 1.031 patients who underwent oocyte retrieval in the IVF unit of our hospital for complications developed during and after the procedure.

Results: No complications developed related to sedation or general anaesthesia. Vaginal bleeding was observed in $3.1 \%$ of the patients. There was no intra-abdominal bleeding or pelvic organ injuries requiring surgery. Two patients developed pelvic abscesses. Ovarian hyperstimulation syndrome (OHSS) occurred in $1.45 \%$ of the patients. Almost all of the patients tolerated the oocyte retrieval process well. After the procedure, only $2 \%$ of the patients described their pain as severe, and $0.4 \%$ as the worst pain they had ever experienced.

Conclusions: The most common complication during oocyte retrieval is vaginal bleeding, which is largely controlled by buffer application. In conclusion, the oocyte retrieval process can be considered a safe procedure.
\end{abstract}

Key words: In vitro fertilisation, oocyte retrieval , post-OR complications

Ginekologia Polska 2018; 89, 1: 1-6

\section{INTRODUCTION}

Even though oocyte retrieval (oocyte pick-up, OPU) comprises a relatively small proportion of the lengthy in vitro fertilisation (IVF) treatment process, it is important to perform the procedure with the correct technique to maintain the maximum oocyte count and avoid potentially life-threatening complications. In this context, the OPU procedure is critical for successful IVF treatment outcomes.

Oocyte collection involves aspirating the fluid-filled ovarian follicles that contain eggs through a needle adapted for transvaginal ultrasonography (TVUSG) probes. Whereas oocyte collection has been performed laparoscopically in the past, transvaginal ultrasound-guided oocyte aspiration under intravenous sedation is now the standard technique [1]. This procedure was first described by Wickland et al. [2] in 1985 and is widely used today in the practice of assisted reproductive techniques $[3,4]$. This simple, effective, and popular method represents the gold standard technique. However, despite these advantages, surgery- or infection-related complications may occur during OPU. The most common complication is bleeding from the lateral fornix through which the OPU needle passes. In addition, complications may occur due to injury to the pelvic organs and vessels caused by the aspiration needle, infection occurring duration the treatment process, or anaesthesia [5-10]. Because many of the complications related to this procedure are underreported, it is not possible to present accurate data about the exact incidence of complications.

\section{OBJECTIVES}

The objective of this study was to investigate the complications that developed during and after transvaginal ultrasound-guided oocyte aspiration procedures performed in the IVF unit of our hospital, and to evaluate the pain experienced by patients during the procedure in the context of the literature. 


\section{MATERIAL AND METHODS Patients}

In this study, we evaluated 1.031 patients who were referred to the IVF unit of the Suleymaniye Obstetrics and Gynaecology Training and Research Hospital between October 1, 2005 and April 30, 2008 with diagnoses of primary or secondary infertility, were scheduled for IVF or intracytoplasmic sperm injection (ICSI), and underwent OPU. This study was approved by our institutional training and planning coordination committee, and used a retrospective design. No age restriction was applied in the selection of patients. All of the patients included in the study were contacted via telephone. Patients who could not be contacted were excluded from the study.

\section{Oocyte retrieval procedure}

The patients underwent controlled ovarian hyperstimulation using standard long-term gonadotropin releasing hormone (GnRH) agonist (long protocol), microdose, or antagonist protocols.

Follow-up was performed with serial TvUSG and serum oestradiol (E2) measurements. Ovulation was triggered with 10,000 IU human chorionic gonadotropin (hCG) (Pregnyl, 5,000 IU amp; Organon International, Oss, Netherlands) when follicular size (dominant follicle larger than $18 \mathrm{~mm}$ or at least two follicles $\geq 17 \mathrm{~mm}$ ) and E2 levels were appropriate.

OPU was carried out 34-36 h after hCG administration. It was recommended that the bladder be emptied prior to the procedure, to reduce the potential for bladder injury and facilitate access to the ovaries during transvaginal oocyte retrieval. Because the procedure is usually completed within 5-10 $\mathrm{min}$, it is not necessary to insert a probe.

The patients were placed on the gynaecological examination table in the lithotomy position and covered with sterile disposable covers. The gynaecologist cleansed the entire vulva and vagina with sterile saline while wearing a disposable box apron and sterile powder-free gloves. The vaginal ultrasonic probe was covered with a sterile plastic sheath. Sedoanalgesia was performed using a combination of propofol/diprivan (Astra Zeneca, Istanbul, Turkey), and fentanyl/fentanyl citrate (Abbott Laboratories, Abbott Park, IL, USA). Some patients were all given a paracervical block using $20 \mathrm{~mL}$ 1\% plain lignocaine as is usual practice in our unit. Following insertion of the ultrasound guide for the vaginal probe, the OPU procedure was performed with a No. 16 single-lumen needle, plus a needle with a valve system for flushing (Cook Medical, Inc., Bloomington, IN, USA), under Sedoanalgesia and guidance by USG. The ultrasound equipment was Voluson-I with a 5- to 9-MHz 4D intracavitary probe (GE Healthcare).

Vaginal and/or abdominal bleeding occurring during and after the OPU procedure was recorded. Incidences of bleeding stopped by local compression, or by use of vaginal tamponade or sutures, were recorded. After the OPU procedure, the patients were held under observation for 2. Preventive antibiotics were not used. At discharge, the patients were questioned to rank their amount of pain $2 \mathrm{~h}$ after oocyte aspiration as to whether they experienced pain, and their self-reported degree of pain was recorded using a verbal descriptor scale (VDS) (pain score of 0-5. 0 - no pain; 1 - mild pain; 2 - moderate pain; 3 - severe pain; 4 - very severe pain; 5 - pain was the worst they that had ever felt) $[11,12]$. The presence of fertilisation was assessed 16-18 $\mathrm{h}$ after OPU. All patients underwent embryo transfers on the third day following the OPU procedure.

After the OPU procedure, all patients were administered $600 \mathrm{mg} /$ day intravaginal intramuscular progesterone (Progestan, 100 mg soft capsule; Koçak Ilac, Istanbul, Turkey) and/or intramuscular Crinone (8\% vg gel; (Serono Laboratories, Norwell, MA, USA), plus1, 500 mg IU hCG every 3 days (Pregnyl; Organon International) to provide luteal phase support.

The patients were administered $\beta$ hCG tests 12 days after the transfer. Positive test outcomes were recorded.

Clinical pregnancy was determined as the detection of a foetal heart beat by abdominal sonography 8 weeks after IVF. Miscarriage was defined as the loss of a clinical pregnancy before the 13th week of gestation. The rates of implantation, clinical pregnancy ,miscarriage abortion , ectopic pregnancy were calculated.

Additionally, all patients were contacted by telephone 2 months after OR and asked for complications, hospital admissions or any episodes of unexplained fever

\section{RESULTS}

A total of 1.031 patients who participated in assisted reproductive cycling and underwent OPU were included in this study. The mean age of the patients was $33.92 \pm 6.71$ years, and the mean body mass index (BMI) was $24.63 \pm 6.49 \mathrm{~kg} / \mathrm{m}^{2}$. Of the patients, 583 (56.6\%) had previously experienced operations with laparotomy and/or laparoscopic methods. Embryo transfer was performed in 803 of the 1.031 patients who underwent OPU. Patient ages, BMls, and previous surgical procedures are shown in Table 1.

The mean number of previous IVF attempts among these patients was $2.01 \pm 0.57$ based on medical records. Sedoanalgesia was applied in 1.003 patients, and a local anaesthesia in 28 patients. The mean number of oocytes collected per patient was $8.93 \pm 4.69$, and the mean number of embryos transferred was $2.07 \pm 0.60$. The total number of transferred embryos was 2.413. The total number of implanted embryos was 437. The implantation rate was calculated as $18.1 \%$. ICSI was performed in 982 cycles, and IVF in 49 cycles. The clinical pregnancy rate was $26.9 \%$ (278/1.031) based 
Table 1. Patients and cycles ' characteristics

\begin{tabular}{|c|c|c|}
\hline Parameters & $\mathbf{n}$ & $(\%)$ \\
\hline Oocyte retrievals (n) & 1031 & \\
\hline Age at retrieval (years) & $33.92 \pm 6.71$ & \\
\hline Body mass index $\left[\mathrm{kg} / \mathrm{m}^{2}\right] \mathrm{a}$ & $24.63 \pm 6.49$ & \\
\hline History of abdominal surgery & $583 / 1031$ & $(\% 56.6)$ \\
\hline - Diagnostic laparoscopy & $159 / 1031$ & $(\% 15.4)$ \\
\hline - Operative laparoscopy & $292 / 1031$ & $(\% 28.3)$ \\
\hline - Laparotomy & $44 / 1031$ & $(\% 4.3)$ \\
\hline - Laparotomy + laparoscopy & $88 / 1031$ & $(\% 8.5)$ \\
\hline \multicolumn{3}{|l|}{ Anaesthesia } \\
\hline Sedoanalgesia & 1003 & $(\%$ 97.3) \\
\hline Local anaesthesia & 28 & $(\% 2.7)$ \\
\hline IVF & 49 & $(\% 4.7)$ \\
\hline ICSI & 982 & $(\% 95.2)$ \\
\hline \multicolumn{3}{|l|}{ Cycle outcome } \\
\hline - Number of collected oocytes (n) & $8.93 \pm 4.69$ & \\
\hline - Two PN & $3.46 \pm 2.90$ & \\
\hline - Embryos transferred (n) & $2.07 \pm 0.60$ & \\
\hline - Cumulative embryo transfer count (n) & 2413 & \\
\hline - Cumulative number of implanted & 437 & \\
\hline \multicolumn{3}{|l|}{ Embryos count ( $n)$} \\
\hline Implantation rate (\%) & $437 / 2413$ & $(\%$ 18.1) \\
\hline Clinical pregnancies per & $367 / 1031$ & $(\% 35.6)$ \\
\hline \multicolumn{3}{|l|}{ Transfer } \\
\hline Clinical pregnancy rate & $278 / 1031$ & $(\% 26.9)$ \\
\hline Abortion rate & $64 / 278$ & $(\% 23)$ \\
\hline Ectopic pregnancy rate & $10 / 278$ & $(\% 3.5)$ \\
\hline Ongoing pregnancy rate & 204/1031 & (\% 19.7) \\
\hline
\end{tabular}

ICSI — inkracytoplasmic sperm infertility; IVF — in vitro fertilisation; PN — pronuclear stage; PN: oocytes at the pronuclear stage mean \pm standard deviation

on the number of OPUs performed. Pregnancies resulted in abortion in $28.57 \%$ of cases $(64 / 274)$, while the rate of ectopic pregnancies was $4.64 \%$ (10/274). The rate of continuing pregnancies was $19.55 \%$ (204/1.031) (Tab. 1)

No complications related to anaesthesia developed. Vaginal bleeding occurred in 32 (3.1\%) patients during and after OPU. The bleeding was stopped with local compression in $25(2.4 \%)$ of these patients, while vaginal tamponade was applied in $0.05 \%$ of the patients $(n=5)$ for $2 \mathrm{~h}$. Only $0.2 \%$ of the cases $(n=2)$ required sutures. There were no complications during and after embryo transfer in the patients that required sutures (Tab. 2). Of the patients who experienced OHSS, one patient was hospitalised due to abdominal pain and tenderness. Of the patients included in the study group, $1.45 \%$ (15/1.031) were hospitalised for OHSS and subsequently treated; severe OHSS (ovaries larger than $12 \mathrm{~cm}$, abdominal distension, nausea, vomiting, diarrhoea, rapid weight gain, dyspnoea, haemoconcentration, and coagulation disorders) was detected in five of these patients. Moderate OHSS (abdominal distension, abdominal swelling, mild pain, nausea, ascites detected on USG, and ovaries between 10-12 cm) was found in 10 patients. Thirty-two cases of mild OHSS were followed up as outpatients by treatment with analgesics and fluid replacement. A positive pregnancy was observed in 4 of the severe OHSS cases, 7 of the moderate OHSS cases, and 15 of the mild OHSS cases. No pelvic organ injuries or major vascular injuries were observed in any patient.

Two patients developed abscesses after the OPU procedure. These patients were hospitalised due to abdominal tenderness and fever. No surgery-related complications were observed in one of the patients, who was laparoscopically operated on and underwent intra-abdominal drainage before being discharged 7 days later. The other patient who developed an abscess was treated only with antibio- 
Table 2. Complications during the procedure, hospitalisations occurring within 2 months after and surgery for complications

\begin{tabular}{|c|c|}
\hline Parameters & n \\
\hline Complications from anaesthesia & 0 \\
\hline Vaginal bleeding & $32 / 1031$ (\% 3.1) \\
\hline - Minor (instant pressure _2 min) & $25 / 1031(\% 2.4)$ \\
\hline - Mild(Vaginal Tamponade) $>2 \mathrm{~h}$ & $5 / 1031(\% 0.5)$ \\
\hline $\begin{array}{l}\text { - Severe (need hospitalisation } \\
\text { sutures/surgery) }\end{array}$ & $2 / 1031(\% 0.2)$ \\
\hline $\begin{array}{l}\text { Severe pain requiring hospitalisation (excluding } \\
\text { OHSS) }\end{array}$ & $1 / 1031$ (\% 0.09) \\
\hline Injuries of pelvic organs & 0 \\
\hline Injury of Pelvic vessel & 0 \\
\hline Pelvic abscess & 2/1031 (\% 0.19) \\
\hline Pelvic infections & 8/1031 (\% 0.77) \\
\hline Ureteral lesion & 0 \\
\hline Ureterovaginal fistulas & 0 \\
\hline Injuries of other organs & 0 \\
\hline Unexplained fever & $1 / 1031$ (\% 0.09) \\
\hline OHSS & $47 / 1031(\% 4.55)$ \\
\hline - Severe OHSS & $5 / 1031$ (\% 0.49) \\
\hline - Moderate OHSS & $10 / 1031(\% 0.96)$ \\
\hline - Mild OHSS & $32 / 1031(\% 3.1)$ \\
\hline
\end{tabular}

OHSS - over hyperstimulation syndrome

tics. Eight patients presented to our unit with mild fever and pelvic tenderness, all of which were treated on an outpatient basis with antibiotics (Tab. 2).

A VDS was applied to investigate the pain experienced by the patients following OPU. It was found that $40.7 \%$ $(420 / 1.031)$ of the patients did not feel any pain, $42 \%$ (433/1.031) had mild pain, $15.5 \%$ (160/1.031) had moderate pain, and $1.6 \%(16 / 1031)$ experienced severe pain; $0.4 \%$
(4/1.031) had very severe pain; $0 \%(0 / 1031)$ reported that the pain was the worst they that had ever felt. Pain status and pain scores are shown in Figure 1 . Only $2.1 \%$ of the patients remembered experiencing pain 24-72 hours after OPU.

\section{DISCUSSION}

Although the risk of serious complications due to OPU is quite low, various complications related to the procedure have been reported, including infection; bladder, bowel, appendix, and ureteral injuries; vaginal bleeding at the entrance of the needle; and minimal to massive bleeding due to needle injuries to pelvic veins [5-10].

This retrospective study of 1.031 patients showed that OPU is a safe and well-tolerated procedure. In this study, there were no anaesthetic complications in patients who underwent sedoanalgesia or local anaesthesia. Two studies in the literature reported complications due to anaesthesia performed for OPU. In the first study, conducted by Chiurgate et al. on a series of 542 patients, intensive care was needed for two patients due to bronchospasm, which occurred after general anaesthesia. Chiurgate et al. and considered these two cases as a random incidence [10]. The second complication type, described in a case report by Ayestaran et al. (2000), was cardiac conduction disorder, which occurred in a patient who received paracervical anaesthesia with $400 \mathrm{mg}$ mepivacaine [13]. Aside from these cases, no other anaesthesia-induced complications were found in the literature [14, 15].

The risk of serious complications due to OPU is very low. Limited vaginal bleeding is relatively common (8\%) at the entrance site of the OPU needle; this can often be stopped by compression, although suturing is sometimes required. $[16,17]$. Acute haemorrhage from the ovary, and bleeding or hematomas due to vascular damage in the uterus, ovary, and iliac vein are very rare $(0.04-0.07 \%)$, but may require surgical intervention when they do occur $[16,18]$. Dessole et al.

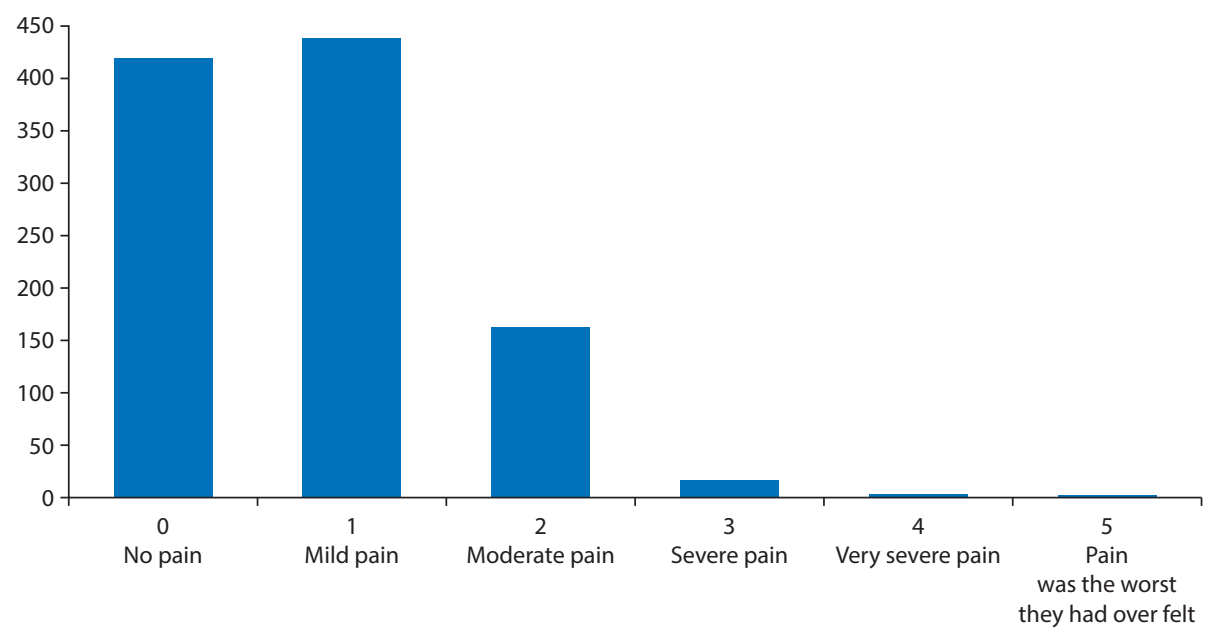

Figure 1. Pain score 
reported mean blood loss after a routine OPU of $230 \mathrm{~mL}$ [19]. Bleeding may be in the form of vaginal, intraperitoneal or retroperitoneal bleeding. Acute intraperitoneal and retroperitoneal bleeding may be recognised during or immediately after TvUSG in the absence of any observable vaginal bleeding, and retroperitoneal bleeding in particular may develop gradually and be diagnosed by USG or other imaging methods after the development of bleeding-induced signs. For example, cases of retroperitoneal hematoma (i.e. the 'Cullen finding'), which have a good course and manifest as periumbilical hematoma, have been reported in the literature [20]. Although massive haemorrhages are mostly due to ovarian, uterine, and iliac vessel injuries, cases of massive retroperitoneal haemorrhage due to injury to the middle sacral vein, and cases of massive haematuria due to bladder pseudoaneurysm haemorrhage, have also been reported [21,22]. Serious bleeding may occur due to clotting disorders [21, 22]. In their studies, Battaglia et al. (2000) and El-Shawarby et al. (2004) reported severe bleeding in two patients with Factor IX deficiency, and in one patient with essential thrombocytopenia [23, 24].

Vaginal bleeding was seen in $3.1 \%$ of our patients and intra-abdominal bleeding was not observed. The bleeding was stopped with local compression in 25 (2.4\%) patients. Vaginal tamponade was applied in $0.05 \%$ of the patients $(n=5)$ for 2 h. Only $0.2 \%(n=2)$ of the cases required sutures. Blood transfusion was not needed in any patient.

Pelvic structures may be traumatised by aspiration needles. Akman et al. and Roest et al. reported perforated appendicitis in two patients after OPU, and the needle hole was observed in these cases $[25,26]$. Contrary to what is believed, intestinal injury is less common, and is more likely to develop in patients with advanced endometriosis, previous surgery, or pelvic adhesions related to previous infections [27]. In our study, no intestinal injuries were observed. Although the intestines and bladder can be seen with ultrasound guidance, injuries to these organs may develop; such injuries may resolve without being noticed and diagnosed. No intestinal or bladder injuries were observed in any of the 1.031 patients in our study, nor were any found on back-questioning of the patients.

Ureteral injury or ureteral obstruction has also been reported. Miller et al., Unsurprisingly, ureteral injuries are underreported, due to the anatomical position of the ureter anterolateral of the upper vaginal fornix [28]. No ureteral injuries were found in our study.

Pelvic infection or pelvic inflammatory disease (PID) may develop after OPU. While the incidence has been reported as $0.02 \%$ by the European Society of Human Reproduction and Embryology data, other studies report an incidence rate of up to $0.3-1.5 \%$ [29-30]. The most likely cause is transfer of vaginal bacterial medium into the peritoneal cavity following perforation of the vagina with the pick-up needle.
In their series of 3.656 patients, Dicker et al. found tubo-ovarian/pelvic abscesses in 99/3.656 (0.24\%) patients. Pelvic infection was controlled through oophorectomy with laparotomy in three, and colpotomy in six, of these patients [18]. In their series of 2.495 patients, Roest et al. detected pelvic infection in six (0.24\%) patients. These six patients were treated with appropriate antibiotics [26]. Askenazi et al. [31] found pelvic infection in 28 of 47.771 patients (0.58\%). All 28 patients were treated with appropriate antibiotics. In our series of 1.031 patients, we found pelvic abscesses in two patients $(0.19 \%)$. These patients were hospitalised due to abdominal tenderness and fever; one of them underwent laparoscopic operation and the abscess was drained. No postoperative complications were observed, and the patient was discharged 7 days later. The other patient who developed an abscess was treated with antibiotics alone. Eight patients were referred to our unit with complaints of mild fever and pelvic tenderness. All of these patients were treated on an outpatient basis with antibiotic therapy.

The pain caused by the OPU procedure was well-tolerated by patients. We observed that the pain increased in direct proportion to the number of oocytes collected and repeated passage through the vaginal fornices. This was due to the high rate of transvaginal access. In their series of 1.166 patients, Ludwig et al. [32] questioned patients about their pain level $2 \mathrm{~h}$ after the OPU procedure.: $16.9 \%$ reported moderate pain, and $3.1 \%$ severe or very severe pain. Furthermore, whereas $7.6 \%$ of patients experienced moderate pain 2 days after OPU, 1.7\% experienced severe or very severe pain. In total, 7 out of 1.166 patients required hospitalisation due to severe pain [32]. In our study, 40.7\% (420/1.031) of patients did not experience any pain, 42\% (433/1.031) experienced mild pain, $15.5 \%$ (160/1.031) moderate pain, $1.6 \%(16 / 1.031)$ severe pain, and $0.4 \%(4 / 1.031)$ reported that the pain was the worst they had ever experienced. Only $2.1 \%$ of patients, all of whom were contacted via telephone, remembered experiencing pain 24-72 h after OPU.

\section{CONCLUSIONS}

Although the OPU procedure is minimally invasive, the risks of pain and bleeding should be considered, and necessary precautions taken. Complications may be recognised immediately due to acute events, or they may be diagnosed later by evaluating patients for latent problems. Therefore, these conditions should be kept in mind in the early follow-up period between oocyte retrieval and embryo transfer and, in later follow-ups, careful attention should be given to potentially unrecognised injuries and their consequences. Patients should be informed about early signs of complications that may develop later.

\section{Acknowledgments}

None. 


\section{REFERENCES}

1. Ditkoff EC, Plumb J, Selick A, et al. Anesthesia practices in the United States common to in vitro fertilization (IVF) centers. J Assist Reprod Genet. 1997; 14(3): 145-147, indexed in Pubmed: 9090556.

2. Wickland M, Enk L, Hamberger L. Transvesical and Transvaginal Approaches for the Aspiration of Follicles by Use of Ultrasound. Ann N Y Acad Sci. 1985; 442: 182-194, doi: 10.1111/j.1749-6632.1985.tb37519.x.

3. Wikland $M$, Enk L, Hammarberg $K$, et al. Use of a vaginal transducer for oocyte retrieval in an IVF/ET program. J Clin Ultrasound. 1987; 15(4): 245-251, indexed in Pubmed: 3134424.

4. Hammarberg K, Enk L, Nilsson L, et al. Oocyte retrieval under the guidance of a vaginal transducer: evaluation of patient acceptance. Hum Reprod. 1987; 2(6): 487-490, indexed in Pubmed: 3667905.

5. Ludwig AK, Glawatz M, Griesinger G, et al. Perioperative and post-operative complications of transvaginal ultrasound-guided oocyte retrieval: prospective study of $>1000$ oocyte retrievals. Hum Reprod. 2006; 21(12): 3235-3240, doi: 10.1093/humrep/del278, indexed in Pubmed: 16877373.

6. Bennett $\mathrm{S}$, Waterstone J, Cheng W, et al. Complications of transvaginal ultrasound-directed follicle aspiration: A review of 2670 consecutive procedures. J Assist Reprod Genet. 1993; 10(1): 7772-7778, doi: 10.1007/bf01204444.

7. Govaerts I, Devreker F, Delbaere A, et al. Short-term medical complications of 1500 oocyte retrievals for in vitro fertilization and embryo transfer. Eur J Obstet Gynecol Reprod Biol. 1998; 77(2): 239-243, indexed in Pubmed: 9578285.

8. El-Shawarby S, Margara R, Trew G, et al. A review of complications following transvaginal oocyte retrieval for in-vitro fertilization. Hum Fertil (Camb). 2004; 7(2): 127-133, doi: 10.1080/14647270410001699081, indexed in Pubmed: 15223762.

9. Seyhan A, Ata B, Son WY, et al. Comparison of complication rates and pain scores after transvaginal ultrasound-guided oocyte pickup procedures for in vitro maturation and in vitro fertilization cycles. Fertil Steril. 2014; 101(3): 705-709, doi: 10.1016/j.fertnstert.2013.12.011, indexed in Pubmed: 24424363.

10. Siristatidis C, Chrelias C, Alexiou A, et al. Clinical complications after transvaginal oocyte retrieval: a retrospective analysis. J Obstet Gynaecol. 2013; 33(1): 64-66, doi: 10.3109/01443615.2012.721818, indexed in Pubmed: 23259882.

11. Ağrı değerlendirme yöntemleri. In: Güzelemir E. ed. Ağrı ve Te-davisi. Ankara : 11-21.

12. Williamson $A$, Hoggart B. Pain: a review of three commonly used pain rating scales. J Clin Nurs. 2005; 14(7): 798-804, doi: 10.1111/j.1365-2702.2005.01121.x, indexed in Pubmed: 16000093.

13. Ayestaran C, Matorras R, Gomez S, et al. Severe bradycardia and bradypnea following vaginal oocyte retrieval: a possible toxic effect of paracervical mepivacaine. Eur J Obstet Gynecol Reprod Biol. 2000; 91(1): 71-73, indexed in Pubmed: 10817882.

14. Andersen AN, Gianaroli L, Felberbaum R, et al. European IVF-monitoring programme (EIM), European Society of Human Reproduction and Embryology (ESHRE). Assisted reproductive technology in Europe, 2001. Results generated from European registers by ESHRE. Hum Reprod. 2005; 20(5): 1158-1176, doi: 10.1093/humrep/deh755, indexed in Pubmed: 15665021.

15. Aragona C, Mohamed MA, Espinola MS, et al. Clinical complications after transvaginal oocyte retrieval in 7,098 IVF cycles. Fertil Steril. 2011; 95(1): 293-294, doi: 10.1016/j.fertnstert.2010.07.1054, indexed in Pubmed: 20727520
16. Ragni G, Scarduelli C, Calanna G, et al. Blood loss during transvaginal oocyte retrieval. Gynecol Obstet Invest. 2009; 67(1): 32-35, doi: 10.1159/000158649, indexed in Pubmed: 18827490.

17. Bennett S, Waterstone J, Parsons J, et al. Two cases of cervical pregnancy following in vitro fertilization and embryo transfer to the lower uterine cavity. J Assist Reprod Genet. 1993; 10(1): 100-103, indexed in Pubmed: 8499672.

18. Dicker D, Ashkenazi J, Feldberg D, et al. Severe abdominal complications after transvaginal ultrasonographically guided retrieval of oocytes for in vitro fertilization and embryo transfer. Fertility and Sterility. 1993; 59(6): 1313-1315, doi: 10.1016/s0015-0282(16)55997-4.

19. Dessole S, Rubattu G, Ambrosini G, et al. Blood loss following noncomplicated transvaginal oocyte retrieval for in vitro fertilization. Fertil Steril. 2001; 76(1): 205-206, indexed in Pubmed: 11438346.

20. Bentov $Y$, Levitas $E$, Silberstein T, et al. Cullen's sign following ultrasound-guided transvaginal oocyte retrieval. Fertil Steril. 2006; 85(1): 227, doi: 10.1016/j.fertnstert.2005.06.054, indexed in Pubmed: 16412762.

21. Jayakrishnan K, Raman VK, Vijayalakshmi VK, et al. Massive hematuria with hemodynamic instability - complication of oocyte retrieval. Fertil Steril. 2011; 96(1): e22-e24, doi: 10.1016/j.fertnstert.2011.04.046, indexed in Pubmed: 21600575.

22. Bergh T, Lundkvist O. Clinical complications during in-vitro fertilization treatment. Hum Reprod. 1992; 7(5): 625-626, indexed in Pubmed: 1639980.

23. Battaglia C, Regnani G, Giulini S, et al. Severe intraabdominal bleeding after transvaginal oocyte retrieval for IVF-ET and coagulation factor XI deficiency: a case report. J Assist Reprod Genet. 2001; 18(3): 178-181, indexed in Pubmed: 11411435.

24. El-Shawarby SA, Margara RA, Trew GH, et al. Thrombocythemia and hemoperitoneum after transvaginal oocyte retrieval for in vitro fertilization. Fertil Steril. 2004; 82(3): 735-737, doi: 10.1016/j.fertnstert.2004.01.044, indexed in Pubmed: 15374723.

25. Akman MA, Katz E, Damewood MD, et al. Perforated appendicitis and ectopic pregnancy following in-vitro fertilization. Hum Reprod. 1995; 10(12): 3325-3326, indexed in Pubmed: 8822469.

26. Roest J, Mous HV, Zeilmaker GH, et al. The incidence of major clinical complications in a Dutch transport IVF programme. Hum Reprod Update. 1996; 2(4): 345-353, indexed in Pubmed: 9080231.

27. Bergh $T$, Lundkvist $O$. Clinical complications during in-vitro fertilization treatment. Hum Reprod. 1992; 7(5): 625-626, indexed in Pubmed: 1639980.

28. Miller PB. Acute ureteral obstruction following transvaginal oocyte retrieval for IVF: Case report. Hum Reprod. 2002; 17(1): 137-138, doi: 10.1093/humrep/17.1.137.

29. Sarhan A. Surgical complications of in vitro fertilization. Middle East Fertility Society Journal. 2007; 12(1): 1-7.

30. Assisted reproductive technology in Europe, 2001. Results generated from European registers by ESHRE. Hum Reprod. 2005; 20(5): 1158-1176, doi: 10.1093/humrep/deh755.

31. Ashkenazi J, Farhi J, Dicker D, et al. Acute pelvic inflammatory disease after oocyte retrieval: adverse effects on the results of implantation. Fertil Steril. 1994; 61(3): 526-528, indexed in Pubmed: 8137978.

32. Ludwig AK, Glawatz M, Griesinger G, et al. Perioperative and post-operative complications of transvaginal ultrasound-guided oocyte retrieval: prospective study of $>1000$ oocyte retrievals. Hum Reprod. 2006; 21(12): 3235-3240, doi: 10.1093/humrep/del278, indexed in Pubmed: 16877373. 